\title{
DNA methylation changes in Down syndrome derived neural iPSCs uncover co- dysregulation of ZNF and HOX3 families of transcription factors
}

Loora Laan ${ }^{1 \dagger}$, Joakim $\mathrm{Klar}^{1 \dagger}$, Maria Sobol', Jan Hoeber ${ }^{1}$, Mansoureh Shahsavani ${ }^{2}$, Malin Kele², Ambrin Fatima ${ }^{1}$, Muhammad Zakaria', Göran Annerén ${ }^{1}$, Anna Falk², Jens Schuster ${ }^{1}$ and Niklas Dahl ${ }^{1 *}$ (D)

\begin{abstract}
Background: Down syndrome (DS) is characterized by neurodevelopmental abnormalities caused by partial or complete trisomy of human chromosome 21 (T21). Analysis of Down syndrome brain specimens has shown global epigenetic and transcriptional changes but their interplay during early neurogenesis remains largely unknown. We differentiated induced pluripotent stem cells (iPSCs) established from two DS patients with complete T21 and matched euploid donors into two distinct neural stages corresponding to early- and mid-gestational ages.

Results: Using the Illumina Infinium 450K array, we assessed the DNA methylation pattern of known CpG regions and promoters across the genome in trisomic neural iPSC derivatives, and we identified a total of 500 stably and differentially methylated CpGs that were annotated to CpG islands of 151 genes. The genes were enriched within the DNA binding category, uncovering 37 factors of importance for transcriptional regulation and chromatin structure. In particular, we observed regional epigenetic changes of the transcription factor genes ZNF69, ZNF700 and ZNF763 as well as the HOXA3, HOXB3 and HOXD3 genes. A similar clustering of differential methylation was found in the CPG islands of the HIST1 genes suggesting effects on chromatin remodeling.

Conclusions: The study shows that early established differential methylation in neural iPSC derivatives with T21 are associated with a set of genes relevant for DS brain development, providing a novel framework for further studies on epigenetic changes and transcriptional dysregulation during T21 neurogenesis.
\end{abstract}

Keywords: Down syndrome, Induced pluripotent stem cells, DNA-methylation, Neurogenesis, Transcription factors, Gene expression

\section{Background}

Trisomy for chromosome 21 (T21) causes Down syndrome (DS) and is a leading cause of intellectual disability with an incidence of approximately 1 in 750 live births [1]. The supernumerary chromosome 21 causes major regional brain abnormalities and a broad range of distinct clinical features [2-4]. We and others have previously shown global gene expression changes in neural cells and brain specimens with T21 [5-10]

\footnotetext{
* Correspondence: niklas.dahl@igp.uu.se

${ }^{\dagger}$ Loora Laan and Joakim Klar contributed equally to this study.

'Department of Immunology, Genetics and Pathology, Science for Life Laboratory, Uppsala University, Box 815, SE-751 08 Uppsala, Sweden Full list of author information is available at the end of the article
}

and it is now generally accepted that DS is the result of complex transcriptomic changes induced by a genomic imbalance of human chromosome 21 (HSA21) [5-10]. While these earlier reports have shown several dysregulated genes in neural cells and brain tissues with T21, the precise mechanisms mediating the genome-wide transcriptomic alterations are still largely unknown.

Methylation of DNA constitutes a potent epigenetic regulatory mechanism for gene expression of fundamental importance for normal embryonic development and for postnatal health [11]. Methylation of promoter regions is associated with condensed inactive chromatin

(c) The Author(s). 2020 Open Access This article is distributed under the terms of the Creative Commons Attribution 4.0 International License (http://creativecommons.org/licenses/by/4.0/), which permits unrestricted use, distribution, and 
and gene silencing whereas methylation within the body of genes may be correlated with splice site usage and increased gene expression [12, 13]. Because the anatomical and cellular brain abnormalities in DS are established at birth, the identification of mechanisms and pathways along the differentiation of trisomic neural cells are important. Prior studies of DNA-methylation patterns in pre- and postnatal brain specimens with T21, as well as in orthologous mice models, have shown alterations across the entire genome when compared to matched euploid tissues [14-16]. Slight generalized hypermethylation in DS fetal cortex has been reported $[14,15]$ accompanied by overexpression of the DNA methyltransferase $3 \mathrm{~L}$, encoded on chromosome 21 [15]. A more recent study, performed by whole-genome bisulfite-sequencing on adult DS brain specimens, reported no differences in global methylation profiles when compared to controls. However, the same study showed enrichment for differentially CpG islands in DS brain samples [17]. While these independent studies have brought important knowledge on epigenetic changes in developing and adult DS brains, the correlation to the global transcriptional changes remains elusive.

The limited access to brain specimens has made neural derivatives from induced pluripotent stem cells (iPSCs) an attractive in vitro model of disease [18]. To date, one study has undertaken a DNA methylation analysis of iPSC with T21 [19] showing methylation differences when comparing trisomic and euploid iPSCs. However, the study was performed on undifferentiated iPSCs and thus not translatable to the developing brain. Noteworthy, the study confirmed enrichment for a subset of differentially methylated $H O X$ genes in trisomic cells, a finding previously observed in the placenta, leukocytes, and buccal cells with T21 [20-23]. Herein, we set out to analyze the methylation pattern of all known CpG regions and promoters in trisomic and matched euploid iPSCs differentiated into the neural lineage. The iPSCderived neural model used in this study has shown a transcriptional profile comparable to that of fetal brains at the early and mid-gestational stages, respectively [10]. We present herein the identification of CpGs regions and promoters across the genome with a consistent pattern of differential methylation pattern in T21 neural cells at two distinct stages of differentiation when compared to euploid cells. Further analysis of differentially methylated CpGs assigned to CpG islands (CGIs) uncovered enrichment of genes for DNA binding and transcriptional regulation. Our study shows the utility of iPSCs derivatives to bring insights into epigenetic mechanisms associated with transcriptional changes during T21 neurogenesis and the combined data provide a framework for further functional studies to interfere with DS brain development.

\section{Results}

Neural iPSCs derivatives with T21 show differentially methylated $\mathrm{CpGs}$ with uneven chromosomal distribution and hypomethylation of chromosome 21

Genomic DNA for methylation analysis of known CpG regions and promoters across the genome was isolated from previously established neural iPSC cultures derived from one male and one female (DS1 and DS2) with characteristic DS features and a full T21, as well as from two age and gender-matched euploid donors (Ctrl1 and Ctrl2) [10]. The DNA was obtained from iPSC derived neural progenitor cells (NPCs) [24], and further differentiated for 30 days (DiffNPC) using an undirected protocol ([10]; Fig. 1). Staining and RNA sequence analysis of neural markers confirmed that the composition of major neural cell types was comparable in trisomic and euploid cultures, and at both differentiation stages (Additional file 1a, b). The transcriptional profiles at the NPC and DiffNPC stages correspond to that of different brain regions, including the hind- and midbrain, at early- and mid-gestation, respectively [10].

Previous studies of the fetal and adult cortex and cerebellum have indicated that at least $90 \%$ of CpGs are stably established at an early fetal stage $[25,26]$. We therefore asked what CpGs exhibited a consistent differential methylation pattern in trisomic NPCs and trisomic DiffNPCs $(n=4)$ when compared to euploid NPCs and DiffNPCs $(n=4)$. To this end, we analyzed the DNA methylation signatures of all known $\mathrm{CpG}$ regions and promoters using the Illumina Infinium Human Methylation $450 \mathrm{~K}$ Bead chip that interrogates $>480,000 \mathrm{CpG}$ sites throughout the genome [27]. The probe call rate for the eight samples analyzed in our study varied between $99.64 \%$ and $99.97 \%$ (detection $p$ value $<0.01$ ). We retrieved $\beta$ values (ratio of intensities between methylated and unmethylated alleles, range between 0 and 1) for 485,512 probes out of a total of 485,553 loci analyzed in all samples [27]. From the raw data of each sample, we prepared density plots of the $\beta$ values for visual inspection (Additional file 2a). Quality assessment of the refined number of probes revealed a bimodal distribution of acquired data (Additional file $2 b$ ) consistent with previous reports $[15,28]$. We then compared the assessed methylation data of trisomic NPCs and DiffNPCs $(n=4)$ with those of euploid NPCs and DiffNPCs $(n=4)$, respectively. Using this approach, we identified 500 differentially methylated probes (DMPs), corresponding to approximately $0.1 \%$ of all CpGs queried by the array, that clustered together in the four trisomic samples at both stages of neural differentiation (Additional file 3). This suggests only small differences in global methylation between the trisomic and euploid neural iPSC derivatives. Hierarchical clustering analysis further illustrated that the differential methylation 


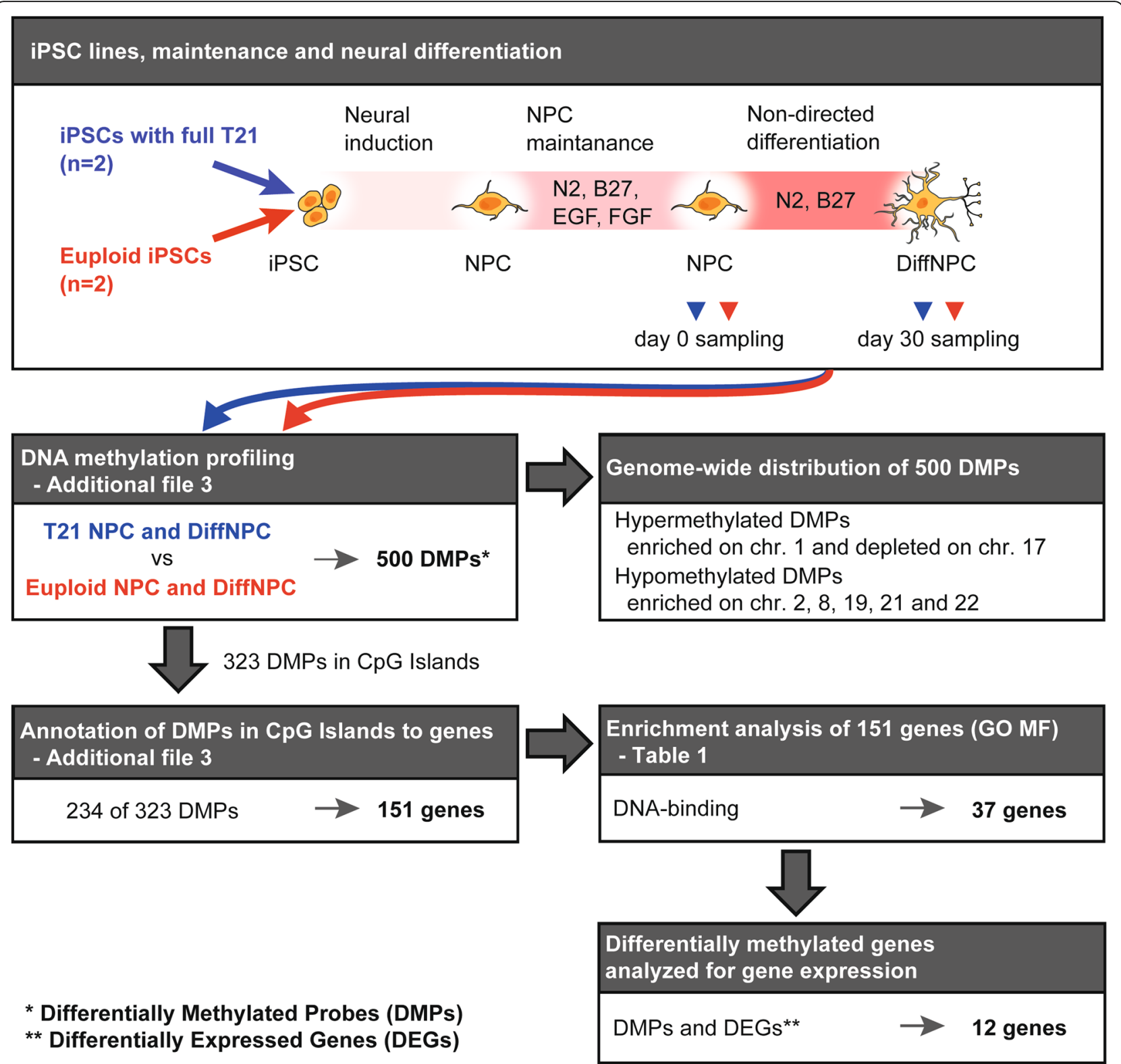

Fig. 1 Overview of the study. Neural iPSC derivatives from two Down syndrome subjects with full trisomy 21 (T21) and two healthy (euploid) subjects were harvested at two stages of differentiation for DNA-methylation analysis of CpGs queried by probes on the 450K array (IIlumina). Differentially methylated probes (DMPs) associated with T21 neural lines, and at two stages of differentiation, were assigned to CpG islands (CGls) and genes. Subsequent enrichment analysis identified 37 genes that were subject to expression analysis

pattern detected by the 500 probes was established during early neural differentiation (in NPCs) and remained into the DiffNPC stage (Fig. 2a). Among the 500 DMPs in trisomic neural cells, 218 (43.6\%) were hypermethylated whereas $282(56.4 \%)$ were hypomethylated $(p=$ 0.049) (Fig. 2b). To further investigate the chromosomal distribution of the CpGs corresponding to the 500 DMPs, we determined the proportion of sites that were hyper- and hypomethylated, respectively, on each chromosome. Notably, several chromosomes in trisomic lines deviated from the expected distribution. The proportion of hypermethylated DMPs were enriched on chromosome 6 but depleted on chromosome 17 ( $p<$ 0.05) (Fig. 2c). On the other hand, chromosomes 2, 8 , 19, 21 and 22 were enriched for hypomethylated sites ( $p$ $<0.05$ ) (Fig. 2d). The enrichment of hypomethylated sites on chromosome 21 is consistent with previous findings in specimens from the fetal DS cortex [14, 15]. These observations suggest that the 500 DMPs, with a stable differential methylation pattern in trisomic NPCs and DiffNPCs, are unevenly distributed on chromosomes. 


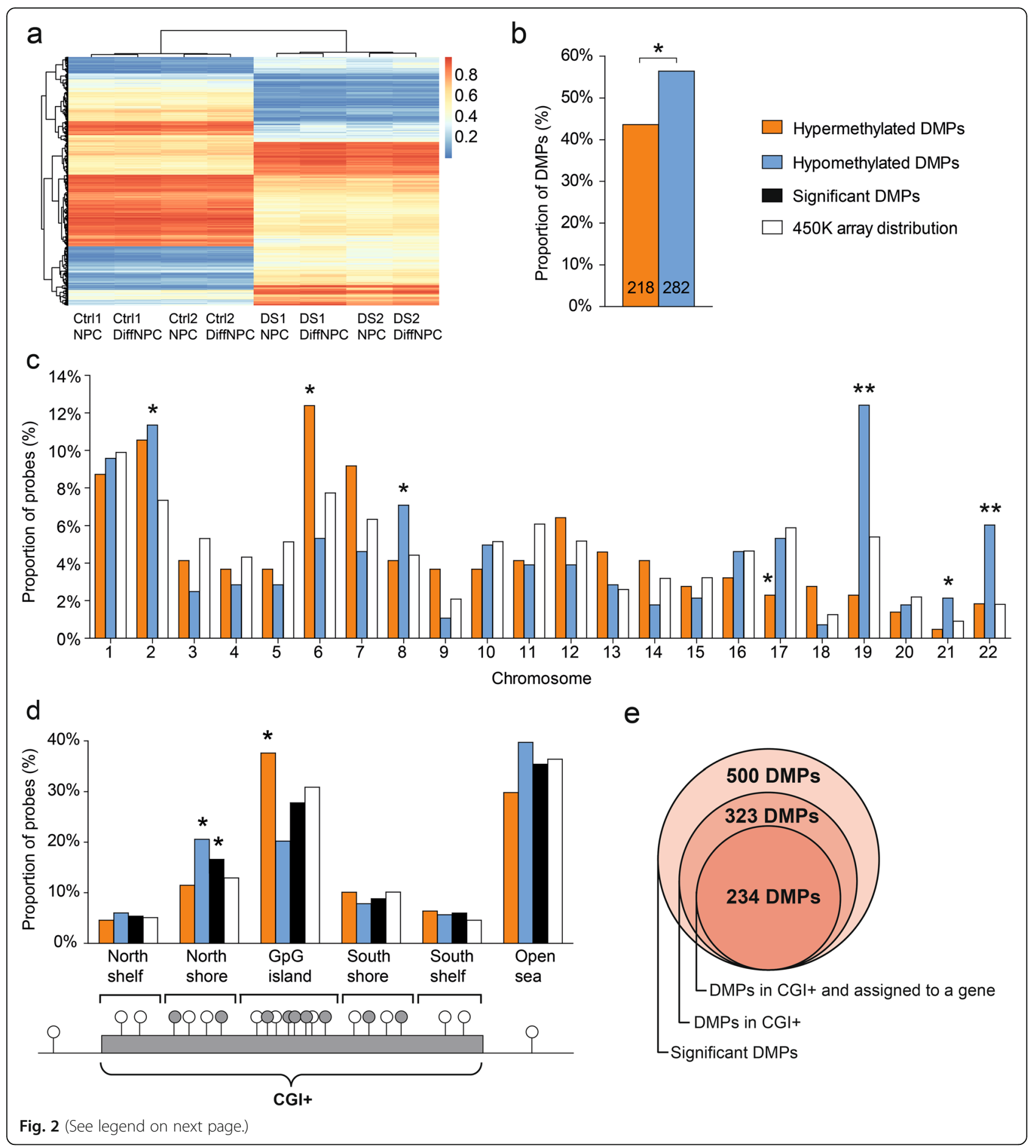


(See figure on previous page.)

Fig. 2 DNA methylation changes in IPSC neural derivatives with T21. a Heat map of 500 differentially methylated CpG sites in T21 and euploid neural cell lines. Hierarchical clustering of the 500 CpGs in euploid (Ctrl) and trisomic (DS) neural lines at the NPC and DiffNPC differentiation stages, respectively (differences $>15 \%$ of average beta values in Ctrl vs. DS lines). Methylation values are color-coded according to legend. $\mathbf{b}$ Bar chart represents the distribution of hypermethylated (orange bars) and hypomethylated DMPs (blue bars) among all 500 DMPs in T21 lines. An enrichment of hypomethylation in T21 neural lines is examined $(p=0.049)$. c Chromosomal distribution of hyper-and hypomethylated DMPs in T21 neural lines. White bars represent percentages of $\mathrm{CpG}$ sites queried on a particular chromosome, orange bars percentages of hypermethylated DMPs and blue bars the percentages of hypomethylated DMPs. Chromosome 1 is significantly $\left(^{*} p<0.05\right)$ enriched for hypermethylated DMPs. Chromosome 17 is depleted of hypermethylated DMPs and chromosomes 2, 8, 19, 21, and 22 are enriched for hypomethylated sites (** $<0.005)$. $\mathbf{d}$ The positions of CpGs called by DMPs are illustrated relative to CpG islands (CGIs) and flanking genomic regions compared to the average of $450 \mathrm{~K}$ array. Bars represent distribution of hypermethylated DMPs (orange; $n=218$ ), hypomethylated DMPs (blue; $n=282$ ), all DMPs (black; $n=500$ ) and 450K array coverage (white) in different genomic regions. Individual CpGs (grey and white circles) were classified based on their location relative to a CpG island (bottom). The proportion of differentially methylated probes in north shores are overrepresented $(p=0.016)$ and preferentially hypomethylated $(p=0.0003)$ when compared to the genome average. In contrast, CpG islands are enriched in hypermethylated CpGs ( $p=0.034)$. e Euler diagram illustrates 500 DMPs in T21 neural iPSCs comprising 323 DMPs located within or close to a $\mathrm{CpG}$ island (CGI+) of which 234 DMPs are assigned to a gene

CpG islands (i.e., CGIs) and their flanking regions usually co-localize with gene promoters and are therefore more likely associated with transcriptional regulation [29-32]. We therefore investigated the methylation pattern of probes annotated close to a CGI, i.e., either within a CGIs [33] or at shores and shelves of a CGI (within $2 \mathrm{~kb}$ or $4 \mathrm{~kb}$ of a CGI, respectively), henceforth defined as a CGI+ (Fig. 2d) [34]. Regions outside a CGI+ are identified as open sea. Out of the 500 DMPs, $323(65 \%)$ were annotated at a CGI+ (Fig. 2d, e). Specifically, 139 (28\%) were located within a CGI; 83 (17\%) in N-shores and $44(9 \%)$ in S-shores; $27(5 \%)$ in $\mathrm{N}$-shelves and $30(6 \%)$ in Sshelves. Out of the 500 DMPs, 177 (35\%) were located in the open sea. The distribution of all 500 DMPs revealed enrichment in $\mathrm{N}$-shores $(p=0.016)$ but was otherwise as expected (Fig. 2d). Furthermore, the DMPs in $\mathrm{N}$-shores were found to be predominantly hypomethylated $(p=0.0003)$. In contrast, DMPs in the CpG islands (CGI) were mainly hypermethylated ( $p=$ 0.034) and with an expected distribution in the total number of DMPs. These data, indicating preferential hypermethylation of CGIs, are consistent with that previously reported in the developing human fetal brain [25]. To corroborate our methylation data from the $450 \mathrm{~K}$ array, we used the bisulfite sequenced-based EpiTYPER assay for region-specific DNA methylation analysis of five CGIs+ called by DMPs [35]. The analysis of the selected CGI+ regions using the EpiTYPER assay confirmed differential methylation in T21 neural lines in all five regions (Additional file $4 \mathrm{a}-\mathrm{c}$ ).

Taken together, we identified 500 DMPs in DNA of trisomic NPCs and DiffNPCs when compared to the corresponding euploid cells. Out of these 500 DMPs, 323 were annotated to within or close to $\mathrm{CpG}$ islands (CGI+) (Fig. 2e, Additional file 5), suggesting a direct regulatory effect.
Differentially methylated genes in neural iPSC derivatives are enriched in the DNA binding category of importance for transcriptional regulation

CGIs are predominantly but not always associated with promoter regions or other regulatory regions of genes [32]. We therefore investigated if the 323 DMPs in CGI+ regions were located close to an annotated gene (i.e., within $1.5 \mathrm{~kb}$ or $200 \mathrm{bp}$ from a transcriptional start site (TSS1500 or TSS200, respectively), in the 5'UTR, first exon, gene body or 3'UTR, of a gene) [36]. This approach uncovered 234 DMPs belonging to a differentially methylated CGI+ region of 151 annotated genes (Fig. 2e). These 151 genes were further analyzed for enrichment in functional categories using the Gene Ontology (GO) knowledge database. Using this approach, we identified a single functional category, namely DNA binding (GO:0003677; Enrichment 2.23; FDR 8.22E-03), that was significantly enriched for 37 genes (Table 1 ). Notably, the DNA binding category comprised multiple members of the HOX, HIST1, and ZNF family of genes encoding proteins important for transcription and chromatin structure (Fig. 3a). Specifically, our analysis identified hypermethylation of $H O X D 4$ and $H O X$ group 3 family members (HOXA3, HOXB3, and $H O X D 3$ ), distributed on chromosomes 2, 7, and 17 (Fig. 3a). The HOX 3 genes are critical for cell fate determination and morphogenesis of several organs such as the brain [37-40]. We also identified hypomethylation of the five histone protein coding genes HIST1H3A, HIST1H4A, HIST1H2BK, HIST1H2AL, and HIST1H3A clustered on chromosome 6 (Fig. 3a, b). The HIST1 genes encode proteins that are critical for chromatin structure and remodeling [41, 42]. Furthermore, out of 13 ZNF genes in the DNA binding category, four were hypomethylated (ZNF69, ZNF441, ZNF700, and ZNF763) and clustered within a $1 \mathrm{Mb}$ region on chromosome 19 (Fig. 3a-c). The downstream targets of these transcription factors 
Table 1 List of 37 enriched genes belonging to the DNA binding category (GO:0003677)

\begin{tabular}{|c|c|c|c|c|c|}
\hline Group & Gene name & CGl position (hg19) & State & \# DMP & DEG \\
\hline \multirow[t]{5}{*}{ HOX proteins } & HOXD4 & chr2:177014948-177015214 & Hyper & 2 & \\
\hline & HOXD3 & chr2:177029413-177029941 & Hyper & 1 & Up \\
\hline & MEISI & chr2:66672431-66673636 & Нyро & 3 & \\
\hline & HOXA3 & chr7:27163819-27164098 & Hyper & 3 & Up \\
\hline & HOXB3 & chr17:46631800-46632212 & Hyper & 1 & Up \\
\hline \multirow[t]{5}{*}{ HIST1 proteins } & HIST1H3A & chr6:26020671-26021125 & Hyper & 1 & \\
\hline & HIST1H4A & chr6:26020671-26021125 & Hyper & 3 & \\
\hline & HIST1H2BK & chr6:27107138-27107394 & Hyper & 1 & \\
\hline & HIST1H2AL & chr6:27833120-27833406 & Hyper & 4 & \\
\hline & HISTIHIB & chr6:27835190-27835461 & Hyper & 1 & \\
\hline \multirow[t]{13}{*}{ ZNF transcription factors } & PRDM16 & chr1:3102540-3103352 & Нyро & 2 & \\
\hline & ZNF512 & chr2:27805754-27806078 & Hyper & 1 & Down \\
\hline & ZNF518B & chr4:10458129-10459353 & Hyper & 1 & Down \\
\hline & CXXC5 & chr5:139040819-139041028 & Нyро & 1 & Up \\
\hline & FEZF1 & chr7:121943867-121944538 & Нyро & 3 & \\
\hline & TRPS1 & chr8:116660432-116660747 & Hyper & 1 & \\
\hline & ZNF263 & chr16:3332472-3333847 & Нуро & 1 & \\
\hline & ZNF397OS & chr18:32847284-32848130 & Hyper & 1 & \\
\hline & ZNF441 & chr19:11877720-11878280 & Нуро & 1 & Up \\
\hline & ZNF69 & chr19:11998804-11999131 & Нyро & 10 & Up \\
\hline & ZNF700 & chr19:12035899-12036433 & Нуро & 9 & Up \\
\hline & ZNF763 & chr19:12076029-12076366 & Нyро & 9 & \\
\hline & ZNF529 & chr19:37095680-37096589 & Hyper & 1 & \\
\hline \multirow[t]{6}{*}{ Transcription factors } & SP5 & chr2:171569877-171573904 & Нyро & 2 & \\
\hline & SP3 & chr2:174828330-174830617 & Нуро & 1 & \\
\hline & NEUROD6 & chr7:31375845-31376542 & Hyper & 1 & \\
\hline & PAX5 & chr9:36985986-36986924 & Hypo & 1 & Up \\
\hline & CREBZF & chr11:85374872-85376234 & Нуро & 1 & \\
\hline & SIM2 & chr21:38079941-38081833 & Нyро & 1 & \\
\hline \multirow[t]{8}{*}{ DNA-binding proteins } & COLECI1 & chr2:3683029-3683290 & Нуро & 1 & \\
\hline & WRNIP1 & chr6:2765203-2766775 & Hyper & 2 & \\
\hline & JRK & chr8:143750759-143751448 & Нyро & 2 & \\
\hline & FBXO21 & chr12:117627650-117628488 & Нуро & 2 & Up \\
\hline & $K D M 2 B$ & chr12:121975028-121976140 & Hyper & 1 & \\
\hline & $P O L E$ & chr12:133249979-133250243 & Нуро & 1 & Down \\
\hline & WNT1 & chr12:49371690-49375550 & Hyper & 2 & \\
\hline & $S M C 1 B$ & chr22:45809191-45809953 & Нуро & 2 & \\
\hline
\end{tabular}

Genes annotated to a CGI+ with at least 1 DMP. Columns denote groups of encoded proteins, position of CGls, methylation state, number of DMPs and differential expression of genes (DEG; Up upregulated, Down downregulated; $p<0.05$ )

(TFs) are yet unknown, however, protein expression data from Human Protein Atlas (HPA version 18.1; proteinatlas.org) [43] show that three of these $Z N F$ genes (ZNF69, ZNF700, and ZNF763) encode for proteins that are highly expressed in glial cells of adult cerebral cortex. Moreover, the differential methylation of the HOX,
HIST1, and ZNF genes is confined to their CGI+ regions with a normal methylation pattern outside the CGI+ regions, further suggesting a regulatory impact (Fig. 3d). Additionally, the DNA-binding category was enriched for six genes (SP5, SP3, NEUROD6, PAX5, CREBZF, and SIM2) encoding TFs of importance for early neural 


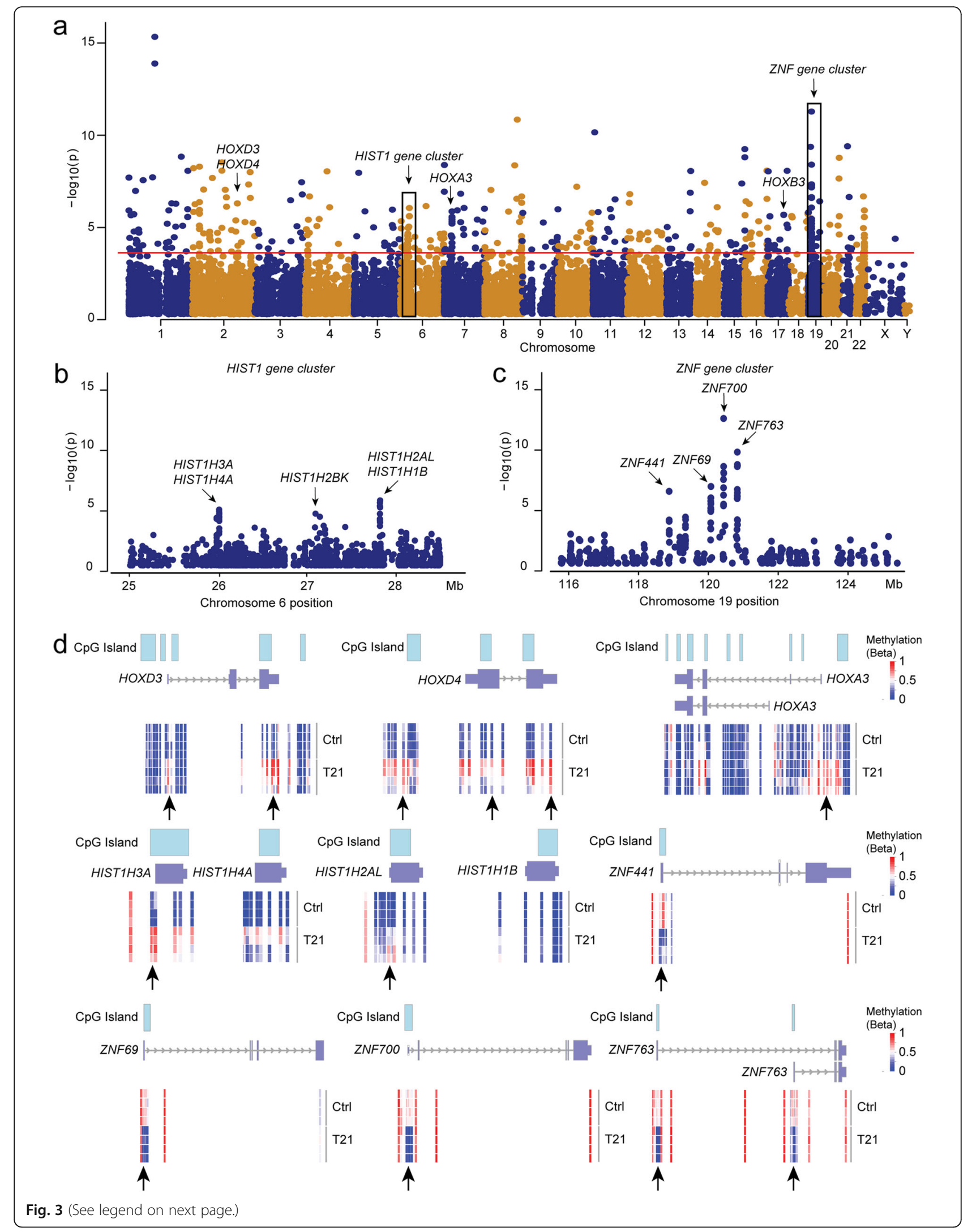


(See figure on previous page.)

Fig. 3 Chromosomal and regional distribution of differential methylation consistent in NPCs and DiffNPCs with T21. a Genomic coordinate dot plot (Manhattan plot) of CpGs detected by 500 DMPs in T21 neural NPCs and DiffNPCs showing methylation changes of known CpG regions and promoters and clustering at annotated genes (arrows and boxes). $X$ axis represents chromosomes ranked by number, $Y$ axis represents - log 10 ( $p$ values) and the red line indicates significance level (Bonferroni; $p<5 \times 10^{-7}$ ). b , c Zoom-in of boxed regions in a showing the hypo-methylated HIST1 gene cluster on chromosome 6 (b) and the hyper-methylated ZNF cluster on chromosome 19 (c). d Details on the DMP pattern (arrows) of three HOX genes (top). Differential methylation of HOXD3 and HOXA3 genes are located in shores and shelves of CGls. In contrast, differential methylation of genes belonging to the HIST1- and ZNF-clusters (mid and bottom) were specific to CGIs. Each DMP is colored according to methylation state ( $\beta$ values) ranging from low (blue) to high (red) methylation in T21 cells

differentiation, as well as a group of eight genes encoding DNA-binding proteins relevant for transcription (Table 1).

As methylation of CGIs and flanking regions are likely to cause transcriptional repression of a nearby gene, we asked if the altered methylation pattern of CGI+ of the 37 enriched genes correlated with differential expression. To this end, we revisited our gene expression data from T21 and euploid lines obtained from the DiffNPC differentiation stage [10]. The analysis revealed that 12 out of the 37 genes (32\%) were differentially expressed $(p<$ 0.05 ) in T21 DiffNPCs (Table 1). To validate the gene expression data retrieved from RNA sequencing, we selected three differentially expressed genes (DEGs; ZNF700, HOXA3, HOXB3) for RT-qPCR analysis that confirmed an altered gene expression (Additional file 6). Notably, three $H O X 3$ genes showed increased expression despite hypermethylated CGI+ regions (Table 1). However, the DMPs in these CGI+ regions were predominantly annotated in shores and not the CGIs themselves.

Taken together, these data show enrichment of differential methylation in CGI+ that are linked to genes encoding for proteins regulating transcription and chromatin remodeling relevant for DS brain development. Given the genome-wide transcriptional perturbations in the DS brain, our data suggest specific epigenetic changes that may contribute to the altered gene expression profile during early neural development in T21 cells.

\section{Discussion}

We present herein, and to our knowledge, the first DNA methylation analysis of neural iPSCs derivatives with T21. When compared to matched euploid cells and previous reports on methylation data obtained from DS brain specimens, the results support our trisomic neural model to be relevant for epigenetic changes in early DS neurogenesis. Despite the low number of biological replicates in our study we identified 500 DMPs, using genomic DNA of neural cells with T21, showing a consistent pattern at two distinct time points of neural differentiation. Importantly, the DMPs were annotated in, or close to, CpG islands of 151 genes enriched in factors important for DNA binding, transcriptional regulation, and chromatin structure.

The 500 DMPs correspond to a small fraction (0.1\%) of all CpGs analyzed. This is in line with a small proportion of DMPs identified in DS cortical samples from previous studies [14, 44]. However, the tendency towards hypomethylation (56\%) of CpGs at the two stages of neural differentiation in our study differs slightly from the tendency towards global hypermethylation observed in fetal and adult DS cortex [14, 15, 17]. Cell typespecific methylation patterns develop already at the neural progenitor stage [45] and cell-specific differences have been identified between glia and neurons that are characteristic for DS cortex [44]. A possible reason for the global increase in hypomethylated DMPs in our iPSC-derived model of DS neurogenesis may thus be due to composition of cell types in the neural cultures. The undirected differentiation protocol leads to a considerable proportion of cells representing mid- and hindbrain precursors [10] that are less abundant in the developing cortex. However, the chromosomal distribution of DMPs in our trisomic neural cells showed a significant enrichment of hypomethylation on chromosome 21 consistent with other studies $[14,15]$. We also identified hypomethylation of chromosomes 2, 8, 19, and 22 whereas chromosomes 6 were enriched and 17 depleted with hypermethylated probes. Uneven chromosomal distribution of DMPs has been observed across a variety of cells and tissues with T21 [14, 15, 46, 47]. The reason for this is unclear and it may be hypothesized that the genomic imbalance in T21 affects the chromosomal organization in $3 \mathrm{D}$, with distorted interactions between certain genomic regions, resulting in a skewing of the chromosomal and subchromosomal DNA methylation pattern.

Among the DMPs, we identified 234 probes called CpGs adjacent to 151 genes and thus with a more likely effect on transcription. This number is comparable to that previously reported for differentially methylated genes in glia and neurons from fetal and adult DS cortical samples [44]. Furthermore, the differentially methylated CGIs in our trisomic neural cells were preferentially hypermethylated consistent with previous studies of the frontal cortex and glia cells in DS brain specimens $[15,17,44]$. Interestingly, we noted that 
several differentially methylated CGI+ regions were clustered close to discrete sets of genes distributed on various chromosomes. These gene sets were further highlighted in our enrichment analysis that uncovered altogether 37 differentially methylated genes within the DNA binding category (GO:0003677). In particular, we uncovered families of genes such as homeobox TFs genes represented by HOXD4, HOXA3, HOXB3, and HOXD3 essential for the development of the hindbrain [37-40] and mesenchymal neural crest-derived structures [48]. Three of these genes showed increased expression in neural T21 cells that were associated with hypermethylation mainly in their shores and shelves (Additional file 3; Fig. 3d). Hypermethylation in shores and shelves around CGIs are sometimes, and in contrast to hypermethylation of CGIs, positively correlated to the expression of genes [49, 50]. Effects on pathways for hindbrain were further supported by differential methylation of the MEIS1 gene encoding a homeobox related TF [51]. In mice, the paralogous Hox3 genes are important for the delineation of the different rhombomeres in the developing hindbrain and for the formation of distinct neuronal lineages within each rhombomere [37]. These observations in our T21 neural lines are consistent with some of the anatomical brain abnormalities in DS such as cerebellar hypoplasia [4, 52]. Furthermore, differential methylation in HOXA3 and/or HOXD3 have previously been observed in glia cells from DS fetal brains [44], peripheral blood leukocytes and fibroblasts of DS patients, and undifferentiated iPSCs with T21 [19, 47] suggesting dysregulated $H O X$ genes to play an important role for a plethora of features characteristic for DS from early stages of development.

Furthermore, we observed hypermethylation of CGIs in the HIST1 gene cluster comprising HIST1H4A, HIST1H3A, HIST1H2BK, HIST1H2AL, and HIST1H1B on chromosome 6 . The cluster constitutes $80 \%$ of genes encoding the canonical histone proteins (H2A, H2B, H3, and $\mathrm{H} 4$ ) and the linker histone (H1) [42] that are of fundamental importance for chromatin structure. Histone modifications regulate the accessibility of chromatin to various TFs and the activity states of DNA [53]. Interestingly, several neurodevelopmental disorders, for example, the ATRX syndrome [54] and Rett syndrome [55], are associated with mutations in genes important for chromatin structure and remodeling. Furthermore, dysregulation of several genes encoding Histone proteins was recently identified in a neurodevelopmental model of Dravet syndrome [56]. Given the role of Histone proteins for chromatin remodeling, differential methylation of the HIST1 gene cluster may suggest a downstream effect on chromatin structure and transcriptional dysregulation in trisomic cells. We also identified differential methylation of 13 genes encoding Zinc-finger transcription factors. Four out of these genes belong to a single cluster on chromosome 19 (Fig. 3a, c) with hypomethylated CGIs. Analysis of RNA sequencing data and by RT-qPCR showed that three of these clustered ZNFgenes had significantly reduced expression levels (Fig. 3d, Table 1) [57]. These Zink-finger TFs are highly expressed in the central nervous system but their precise role during development is yet unknown. Zinc-finger proteins belong to the most abundant class of proteins in the human proteome but the majority remain uncharacterized as well as their downstream targets [58, 59]. However, the ability of zink-finger domains to interact with DNA and RNA suggests this family of proteins have a role in a broad range of functions beside transcriptional regulation. Furthermore, our enrichment analysis identified two additional groups of genes encoding six different transcription factors and eight DNA interacting proteins (Table 1). One of the genes is SIM2 within the DS critical region on chromosome 21 [9] encoding a TF important for cell fate determination [60]. Interestingly, a prior study showed that SIM2 expression in fetal brain co-localizes with regions associated with DS pathology [61]. Taken together, the differentially methylated CGI+ regions identified in our trisomic neural model highlights genes and gene families of importance for transcriptional regulation and chromatin structure, providing further mechanistic insights into DS neurogenesis.

The association of DMPs with genes that are enriched in the DNA-binding category and for transcriptional regulation in our T21 neural model is in line with the previously and well documented global transcriptional changes of the DS brain [8]. It has been proposed that epigenetic mechanisms are critical for the transcriptional perturbations in DS [9] and previous reports have shown enrichment for DMPs at certain TF binding motifs in DS brain specimens [17, 44]. Given the genome-wide gene expression changes in DS brains, the differentially methylated genes and gene families identified in our study suggest important novel links between the genomic unbalance caused by T21 and the global transcriptional dysregulation in DS brains. We recently showed that the transcriptional dysregulation in iPSC-derived neural cells with T21 is confined to major functional clusters [10]. While this previous study suggested major functional clusters to be perturbed in T21 neural cells, DNA-binding was not identified as a disrupted functional category. The present study thus adds to previous reports by showing marked differential methylation confined to a set of genes important for transcriptional regulation and chromatin remodeling. In support of these findings, one-third of the genes enriched in the DNA binding category were found differentially expressed. 
The vast majority of $\mathrm{CpGs}$ in the human genome are predominantly methylated as 5 -methylcytosine $(5 \mathrm{mC}$; methylation at the 5-carbon position) [11]. Hydroxylation of $5 \mathrm{mC}$ results in $5 \mathrm{hmC}$, a demethylation intermediate shown to be present in $4 \%$ of mammalian CpGs $[62,63]$. It has been suggested that $5 \mathrm{hmC}$ may be a regulator for the elimination of cytosine methylation during development [63, 64]. Unfortunately, the commonly used methods to study DNA methylation based on bisulfite treatment do not distinguish between $5 \mathrm{mC}$ and $5 \mathrm{hmC}$ [62] why different detection methods are required to resolve the potential regulatory role of $5 \mathrm{hmC}$ in neurogenesis.

Intellectual disability is a predominant feature in DS and likely related to several distinct cell populations. The human brain forms highly diverse neuronal subpopulations presumably characterized by distinct methylation and expression profiles. Indeed, it has been demonstrated that the methylation pattern is different when comparing major neuronal subtypes in both mice [45] and humans [44]. Furthermore, a previous study reported that methylation patterns clustered differently when comparing distinct cortical brain regions as well as when comparing specific brain regions from DS patients with those from healthy subjects [44]. In our neural cultures, the cell populations are mixed with a predominance of mid- and hindbrain neural cells [24]. Given the heterogeneity of neural cells in our culture system, the methylation changes associated with T21 may therefore reflect a sum of different methylation signatures related to different neural subpopulations. Furthermore, our study focused on DMPs showing a consistent pattern at two stages of neural differentiation corresponding to early and mid-gestation. This approach selected for DMPs that are stably established at an early differentiation stage. However, previous studies on fetal brain tissues have shown only minor changes in the overall differential methylation pattern with gestational ages [25, 44] supporting that the majority of DMPs detected in our iPSC derived neural cells with T21 reflects an early established and stable differential methylation pattern.

\section{Conclusions}

Our study suggests that iPSCs with T21 differentiated into neural lineages may serve as a translatable model for the identification of epigenetic changes that are associated with transcriptional perturbations in DS neurogenesis. The differentially methylated regions identified in our trisomic neural model highlights specific genes and gene families of importance for transcriptional regulation and chromatin structure. Taken together, the data provides a framework for further studies on epigenetic variation and specific factors mediating transcriptional changes downstream of chromosome 21 during DS brain formation. While our model shows extensive promise for further understanding of molecular mechanisms behind perturbed and early neurogenesis in DS, the undirected protocol used to generate neural cells from iPSCs is not translatable to the cortex but rather to the entire fetal brain at early stages of development [10]. Further investigations of differential methylation in functionally distinct neuronal cell populations with T21, preferably connected to transcriptome profiles, are now required to clarify the role of DNA-methylation changes for perturbed neurogenesis and ultimately for DS brain formation. More complex models of human neural differentiation using, e.g., 3D organoids from iPSCs with T21, build up by a mixture of cell types that are analyzed individually, may add important information in this context.

\section{Methods}

iPSC lines, maintenance, and neural differentiation

We previously established iPSCs with HSA21 by transducing fibroblast cells from one male and one female (DS1 and DS2) with characteristic DS features and a full T21, as well as from two age and gender-matched healthy individuals (Ctrl1 and Ctrl2) [10]. Fibroblasts were reprogrammed using a non-integrating method with CytoTune ${ }^{\mathrm{TM}}$-iPS 2.0 Sendai Reprogramming Kit (ThermoFisher Scientific, cat no: A16517). Standard karyotype analysis confirmed T21 or euploidy in iPSC derived from DS patients and healthy donors, respectively. Selected iPSC lines were induced to neural progenitor cells (NPC) as described [10]. The NPCs were grown on $0.1 \mathrm{mg}$ poly-L-ornithine (Sigma-Aldrich, cat no.: P4957-50ML) and $1 \mu \mathrm{g} / \mathrm{mL}$ laminin (Sigma-Aldrich, cat no: L2020-1MG)-coated plates in DMEM/F12 GlutaMAX medium (Gibco, cat no.: 31331028) supplemented with $10 \mathrm{ng} / \mathrm{mL}$ rhFGF-basic (R\&D Systems, cat no.: 233FB-010), $10 \mathrm{ng} / \mathrm{mL}$ rhEGF (R\&D Systems, cat no.: 236EG-200), B27 supplement (1:1000, Gibco, cat no.: 080085SA), N2 supplement (1:100, Gibco, cat no.: 17502048), and 1\% penicillin/streptomycin (Gibco, cat no.: 15140-122). Self-renewing NPCs derived from the four donors were differentiated for 30 days into DiffNPCs using an undirected protocol (Fig. 1) as described [10, 24].

\section{Immunofluorescence}

Immunofluorescent staining of the trisomic and euploid neural cultures was performed using standard techniques [65] at both differentiation stages as described previously [10]. The primary antibodies against Nestin (1:100, R\&D Systems, cat no.: MAB1259), Pax6 (1:100, BioLegend, cat no.: 901301), $\beta$-III-tubulin (1:80, SigmaAldrich, cat no.: T2200), GFAP (1:500, Sigma-Aldrich, cat no.: G3893-.2ML), and Vimentin (1:500, Abcam, cat no.: ab92547) were incubated overnight at $4{ }^{\circ} \mathrm{C}$. 
Subsequently, the cells were stained with $\alpha$-mouse IgG Alexa Fluor 488 (1:10000, Thermo Fisher Scientific, cat no.: A-11001) and $\alpha$-rabbit IgG Alexa Fluor 555 (1: 10000, Thermo Fisher Scientific, cat no.: A-21406) for $1.5 \mathrm{~h}$ at room temperature. Nuclei were stained with DAPI (1 $\mu \mathrm{g} / \mathrm{mL}$, Sigma-Aldrich, cat no.: D8417). Visualization was performed on a Zeiss 510 confocal microscope.

\section{Heat-map profiling of neural genes}

A set of known markers for neuroepithelial cells, radial glia, oligodendrocytes, astrocytes, microglia, immature neurons, mature neurons, glutamatergic neurons, and GABAergic neurons were selected. Expression of markers was retrieved and analyzed from RNA sequencing data $(\log 2$ (counts) ) of trisomic and euploid derived neural cultures at the NPC and DiccNPC stages of differentiation [10]. The Pheatmap package in $\mathrm{R}$ was used to generate heatmap profiles of the expressed marker genes.

\section{DNA extraction and microarray analysis}

Total Genomic DNA was isolated using NucleoSpin ${ }^{\bullet}$ Tissue kit (Macherey-Nagel, cat no.: 740952.250) following the manufacturer's instructions. Extracted genomic DNA was treated with sodium bisulfite using EZ-96 DNA methylation Gold kit (Zymo Research, cat no.: D5007). Assessment of levels of DNA methylation of known CpG regions and promoters across the genome was done with Illumina HumanMethylation 450K BeadChip and Illumina HiScan 2000. In brief, following bisulfite conversion, approximately $200 \mathrm{ng}$ of the bisulfiteconverted DNA per sample was used for methylation analysis. The initial quality control and identification of signal intensities for each probe were performed with Illumina GenomeStudio Software.

\section{Data analysis of differential methylation}

The methylation data was analyzed using the Minfi package by a group-wise comparison between T21 and control samples [66]. Beta values were calculated to estimate methylation levels from the ratio of intensities between methylated and unmethylated alleles. Beta values ranged between 0 and 1 , with 0 being unmethylated and 1 fully methylated. Differentially methylated probes (DMPs) were identified with dmpFinder using SWAN normalized values (betaThreshold $>0.1$ ). The data was plotted in a Manhattan plot using the qman package in R. To avoid small methylation differences between T21 and euploid lines due to stochastic variations, we only considered differences of $>15 \%$ (average beta values of patients vs. controls) using an FDR adjusted $p$ value ( $q$ value) and a cut off $<0.05$. To investigate the chromosomal distribution of CpGs detected by DMPs, the number of hypo-and hypermethylated DMPs per chromosome was compared with the total number of $450 \mathrm{~K}$ array probes per respective chromosome. Enrichment or depletion of CpGs with respect to chromosomal location and methylation status were calculated using data on called DMPs and Fisher's exact test (two-sided $p<0.05$ were considered significant).

\section{Bisulfite sequencing}

For verification, we performed targeted analysis of DNA methylation levels of five genes annotated to CGIs with at least two DMPs (EDNRB, ZNF700, HOXA3, GGCT, and $R I B C 2$ ) using the EpiTYPER ${ }^{\text {max }}$ technology (Agena Bioscience). Genomic DNA was treated with sodium bisulfite (EZ-96 DNA Methylation ${ }^{\text {Tx }}$ kit, Zymo Research, cat no.: D5004) followed by PCR using primers designated in the EpiDesigner software (epidesigner.com, Agena Biosciences; Primer sequences available upon request). Targeted regions were amplified with PCR using T7-promoter-tagger reverse primers followed by in vitro transcription. The resulting transcripts were specifically cleaved at uracil residues and subjected to MALDI-TOF analysis on an Agena Compact MassARRAY Analyzer. The EpiTYPER software 1.2.22 was used to identify the mass-fragments and for quantification of DNAmethylation at single- $\mathrm{CpG}$ sites or of a $\mathrm{CpG}$ unit. $\mathrm{CpG}$ sites analyzed from bisulfite-sequencing were compared with methyl-sequenced CpGs. Analysis of differential methylation was calculated using unpaired $t$ test, following the Mann-Whitney $U$ test $(p<0.05$ was considered significant). Bisulfite-sequencing data was also used to generate DNA methylation profiles of five genes followed by two-way ANOVA.

\section{RNA extraction and quantitative RT-PCR}

Total RNA was extracted from cell lysates with the RNeasy ${ }^{\circ}$ Mini Kit (Qiagen, cat no.: 74104) following the manufacturer's instructions. DNase I treated total RNA samples $(1 \mu \mathrm{g})$ were subject to first-strand DNA synthesis by High Capacity cDNA Synthesis kit (ThermoFisher Scientific, cat no.: 4368814). Quantitative RT-qPCR was performed using FastStart Universal SYBR Green Master (Rox) mix (Sigma-Aldrich, cat no.: 4913850001) following manufacturer's protocol. The reactions were performed in duplicates and run on the StepOnePlus ${ }^{\mathrm{Tm}}$ RealTime PCR System (Applied Biosystems). The expression levels of genes were measured using the primers listed below. Primer design was done with Primer3 online tool [67]. The results were normalized against GAPDH. The analysis was performed in StepOne Software v2.2.2 and GraphPad prism and differential expression was calculated using unpaired t-test with Welch's correction ( $p<0.05$ was considered significant). 
The expression levels of the genes were measured using the following primers:

GAPDH F: 5' -GTCAGCTGTTGTTGGACCTG-3', GAPDH R: 5'-GGTCACCCCATCGAAGATAC-3', ZNF700 F: 5'-CACCCAGGAAGAGTGGACAT-3', ZNF700 R: 5' -ATGCCTTGTGTCCAGTGTCA-3' HOXA3 F: 5' - 'TGCCCTTCTGATCCTTTTTG-3', HOXA3 R: 5'-AATGCCAGCAACAACCCTAC-3', HOXB4 F: 5' -CTGGATGCGCAAAGTTCAC-3', HOXB4 R: 5' -AGCGGTTGTAGTGAAATTCCTT-3',

\section{Analysis of genes associated with DMPs}

For gene annotation analysis, we first mapped the CpGs detected by DMPs with respect to CGI+ regions (i.e., positioned in a CGI or in the shores or shelves of that CGI) or to open sea. We thereafter investigated if DMPs in CGI+ regions coincided with an annotated gene (UCSC; genome.ucsc.edu) according to the Illumina manifest (i.e., a CGI+ within $1.5 \mathrm{~kb}$ or $200 \mathrm{bp}$ from a transcriptional start site (TSS1500 or TSS200, respectively), in the 5'UTR, first exon, gene body or 3'UTR, of a gene) [36]. A single gene name was annotated to each DMP using this criterion and if one or more gene name entries were annotated to one DMP the first gene was used in the enrichment analysis (Additional file 3). Probes with no UCSC RefGene name were not included in the analysis. Enrichment in functional categories was performed using the Gene Ontology (GO) knowledgebase database (geneontology.org; PANTHER Overrepresentation Test (Released 20190711)) and the Molecular Function category. Gene expression data was retrieved for DiffNPC differentiation stage from Sobol et al., 2019 [10].

\section{Supplementary information}

Supplementary information accompanies this paper at https://doi.org/10. 1186/s13148-019-0803-1.

Additional file 1. Characterization of neural cultures with trisomy 21 (DS) and euploid controls (Ctrl) at the NPC and DiffNPC differentiation stages.

Additional file 2. Quality control of Illumina 450K array data. Additional file 3. List of all 500 DMPs called in T21 neural lines. Additional file 4. Validation of DNA methylation data from $450 \mathrm{~K}$ array analysis.

Additional file 5. Genes annotated to CGI+ regions that are differentially methylated in T21 neural lines.

Additional file 6. Validation of gene expression levels by RT-qPCR.

\section{Abbreviations}

5mC: 5 methylcytosine; CGl: CpG island; DiffNPC: Differentiated neural progenitor cell;; DMP: Differentially methylated probe; DS: Down syndrome; HSA21: Human chromosome 21; iPSC: Induced pluripotent stem cell; NPC: Neural progenitor cell; T21: Trisomy for chromosome 21;

TF: Transcription factor

\section{Acknowledgements}

We thank Mikael Huss for valuable discussions. The 450K array data were generated at the SNP\&SEQ platform, Science for Life Laboratory, Uppsala University. Computations were performed on resources provided by SNIC through Uppsala Multidisciplinary Center for Advanced Computational Science (UPPMAX).

\section{Authors' contributions}

ND and JK conceptualized and designed the study. LL, JK, MS, JH, SM, MK, $M Z, A F$, and AF contributed to the methodology, acquisition of data, and primary analysis. $L L, J K, M S, J H, J S$, and ND contributed to the formal interpretation. $L L$ and JK wrote the manuscript draft. JK, GA, JS, AF, and ND contributed to the final editing.. JK, JS, AF, and ND supervised the study. All authors read and approved the final version of the manuscript.

\section{Funding}

This work was supported by grants from the Swedish Research Council (2015-02424 to ND), Sävstaholm Society, Astra-Zeneca, Hjärnfonden (FO20180100 and FO2019-0210 to ND), Uppsala University, the Knut and Alice Wallenberg Foundation as part of the National Bioinformatics Infrastructure Sweden at Science for Life Laboratory. L.L. was funded by grants from the Sävstaholm Society and her contribution was independent of support from AstraZeneca. The funders played no role in study design, data collection, and interpretation or decision to publish. Open access funding provided by Uppsala University.

\section{Availability of data and materials}

The datasets supporting the conclusions of this article are included within the article, its additional files and, in Supplementary Table 2 of reference [10]: 12035_2019_1585_MOESM2_ESM.xIs.

\section{Ethics approval and consent to participate}

Written informed consent for publication was obtained from the donors of skin punch biopsies, or by their legal guardians. This study was reviewed and approved by the regional ethical committee of Uppsala, Sweden (Dnr 2016/ 209).

\section{Competing interests}

The authors declare that they have no competing interests.

\section{Author details}

'Department of Immunology, Genetics and Pathology, Science for Life Laboratory, Uppsala University, Box 815, SE-751 08 Uppsala, Sweden.

${ }^{2}$ Department of Neuroscience, Karolinska Institutet, Stockholm, Sweden.

Received: 10 September 2019 Accepted: 23 December 2019

Published online: 08 January 2020

References

1. Parker SE, Mai CT, Canfield MA, Rickard R, Wang Y, Meyer RE, et al. Updated National Birth Prevalence estimates for selected birth defects in the United States, 2004-2006. Birth Defects Res A Clin Mol Teratol. 2010;88:1008-16.

2. Raz N, Torres IJ, Briggs SD, Spencer WD, Thornton AE, Loken WJ, et al. Selective neuroanatomic abnormalities in Down's syndrome and their cognitive correlates: evidence from MRI morphometry. Neurology. 1995;45: 356-66.

3. Guidi S, Bonasoni P, Ceccarelli C, Santini D, Gualtieri F, Ciani E, et al. Neurogenesis impairment and increased cell death reduce total neuron number in the hippocampal region of fetuses with Down syndrome. Brain Pathol. 2008;18:180-97.

4. Haydar TF, Reeves RH. Trisomy 21 and early brain development. Trends Neurosci. 2012;35:81-91.

5. Mao R, Wang X, Spitznagel ELJ, Frelin LP, Ting JC, Ding H, et al. Primary and secondary transcriptional effects in the developing human Down syndrome brain and heart. Genome Biol. 2005;6:R107.

6. Lockstone HE, Harris LW, Swatton JE, Wayland MT, Holland AJ, Bahn S. Gene expression profiling in the adult Down syndrome brain. Genomics. 2007;90: 647-60

7. Letourneau A, Santoni FA, Bonilla X, Sailani MR, Gonzalez D, Kind J, et al. Domains of genome-wide gene expression dysregulation in Down's syndrome. Nature. 2014;508:345-50. 
8. Olmos-Serrano JL, Kang HJ, Tyler WA, Silbereis JC, Cheng F, Zhu Y, et al. Down Syndrome Developmental Brain Transcriptome Reveals Defective Oligodendrocyte Differentiation and Myelination. Neuron. 2016;89:1208-22.

9. Antonarakis SE. Down syndrome and the complexity of genome dosage imbalance. Nat Rev Genet. 2017;18:147-63.

10. Sobol M, Klar J, Laan L, Shahsavani M, Schuster J, Anneren G, et al. Transcriptome and proteome profiling of neural induced pluripotent stem cells from individuals with Down syndrome disclose dynamic dysregulations of key pathways and cellular functions. Mol Neurobiol. 2019;10:7113-27.

11. Smith ZD, Meissner A. DNA methylation: roles in mammalian development. Nat Rev Genet. 2013;14:204-20.

12. Newell-Price J, Clark AJ, King P. DNA methylation and silencing of gene expression. Trends Endocrinol Metab. 2000;11:142-8.

13. Jjingo D, Conley AB, Yi SV, Lunyak W, Jordan IK. On the presence and role of human gene-body DNA methylation. Oncotarget. 2012;3:462-74.

14. El Hajj N, Dittrich M, Böck J, Kraus TFJ, Nanda I, Müller T, et al. Epigenetic dysregulation in the developing Down syndrome cortex. Epigenetics. 2016; 11:563-78

15. Lu J, Mccarter M, Lian G, Esposito G, Capoccia E, Delli-Bovi LC, et al. Global hypermethylation in fetal cortex of Down syndrome due to DNMT3L overexpression. Hum Mol Genet. 2016:25:1714-27.

16. Do C, Xing Z, Yu YE, Tycko B. Trans-acting epigenetic effects of chromosomal aneuploidies: lessons from Down syndrome and mouse models. Epigenomics. 2017;9:189-207.

17. Laufer BI, Hwang H, Vogel Ciernia A, Mordaunt CE, LaSalle JM. Whole genome bisulfite sequencing of Down syndrome brain reveals regional DNA hypermethylation and novel disorder insights. Epigenetics. 2019;14: 672-84.

18. Dolmetsch $\mathrm{R}$, Geschwind $\mathrm{DH}$. The human brain in a dish: the promise of iPSC-derived neurons. Cell. 2011;145:831-4.

19. Sailani MR, Santoni FA, Letourneau A, Borel C, Makrythanasis P, Hibaoui $Y$, et al. DNA-methylation patterns in Trisomy 21 using cells from monozygotic twins. PLoS One. 2015;10:e0135555.

20. Jin S, Lee YK, Lim YC, Zheng Z, Lin XM, Ng DP, et al. Global DNA Hypermethylation in Down Syndrome Placenta. PLoS Genet. 2013;9: e1003515.

21. Malinge S, Chlon T, Dore LC, Ketterling RP, Tallman MS, Paietta E, et al. Development of acute megakaryoblastic leukemia in Down syndrome is associated with sequential epigenetic changes. Blood. 2013;122:e33-43.

22. Jones MJ, Farré $P$, McEwen LM, Macisaac JL, Watt K, Neumann SM, et al. Distinct DNA methylation patterns of cognitive impairment and trisomy 2 in down syndrome. BMC Med Genomics. 2013;6:58.

23. Kerkel K, Schupf N, Hatta K, Pang D, Salas M, Kratz A, et al. Altered DNA methylation in leukocytes with trisomy 21. PLoS Genet. 2010;6:e1001212.

24. Falk A, Koch P, Kesavan J, Takashima Y, Ladewig J, Alexander M, et al. Capture of neuroepithelial-like stem cells from pluripotent stem cells provides a versatile system for in vitro production of human neurons. PLoS One. 2012;7:1-13.

25. Spiers H, Hannon E, Schalkwyk LC, Smith R, Wong CCY, O'Donovan MC, et al. Methylomic trajectories across human fetal brain development. Genome Res. 2015;25:338-52

26. Schneider E, Dittrich M, Bock J, Nanda I, Muller T, Seidmann L, et al. CpG sites with continuously increasing or decreasing methylation from early to late human fetal brain development. Gene. 2016;592:110-8.

27. Bibikova M, Barnes B, Tsan C, Ho V, Klotzle B, Le JM, et al. High density DNA methylation array with single CpG site resolution. Genomics. 2011;98:288-95.

28. Slieker RC, Bos SD, Goeman JJ, Bovee JV, Talens RP, van der Breggen R, et al. Identification and systematic annotation of tissue-specific differentially methylated regions using the Illumina 450k array. Epigenetics Chromatin. 2013;6:26.

29. Jones PA. Functions of DNA methylation: islands, start sites, gene bodies and beyond. Nat Rev Genet. 2012;13:484-92.

30. Deaton AM, Bird A. CpG islands and the regulation of transcription. Genes Dev. 2011:25:1010-22.

31. Ziller MJ, Gu H, Muller F, Donaghey J, Tsai LT-Y, Kohlbacher O, et al. Charting a dynamic DNA methylation landscape of the human genome. Nature. 2013;500:477-81.

32. loshikhes IP, Zhang MQ. Large-scale human promoter mapping using $\mathrm{CpG}$ islands. Nat Genet. 2000;26:61-3.

33. Gardiner-Garden M, Frommer M. CpG islands in vertebrate genomes. J Mo Biol. 1987;196:261-82.
34. Sandoval J, Heyn H, Moran S, Serra-Musach J, Pujana MA, Bibikova M, et al. Validation of a DNA methylation microarray for 450,000 CpG sites in the human genome. Epigenetics. 2011;6:692-702.

35. Suchiman HED, Slieker RC, Kremer D, Slagboom PE, Heijmans BT, Tobi EW. Design, measurement and processing of region-specific DNA methylation assays: the mass spectrometry-based method EpiTYPER. Front Genet. 2015;6: 287.

36. Kent WJ, Sugnet CW, Furey TS, Roskin KM, Pringle TH, Zahler AM, et al. The human genome browser at UCSC. Genome Res. 2002;12:996-1006.

37. Gaufo GO, Thomas KR, Capecchi MR. Hox3 genes coordinate mechanisms of genetic suppression and activation in the generation of branchial and somatic motoneurons. Development. 2003;130:5191-201.

38. Deschamps J, van Nes J. Developmental regulation of the Hox genes during axial morphogenesis in the mouse. Development. 2005;132:2931-42.

39. Parker HJ, Bronner ME, Krumlauf R. The vertebrate Hox gene regulatory network for hindbrain segmentation: Evolution and diversification: Coupling of a Hox gene regulatory network to hindbrain segmentation is an ancient trait originating at the base of vertebrates. Bioessays. 2016;38:526-38.

40. Morrison A, Ariza-McNaughton L, Gould A, Featherstone M, Krumlauf R. HOXD4 and regulation of the group 4 paralog genes. Development. 1997; 124:3135-46.

41. Thoma F, Koller T, Klug A. Involvement of histone $\mathrm{H} 1$ in the organization of the nucleosome and of the salt-dependent superstructures of chromatin. J Cell Biol. 1979;83:403-27.

42. Albig W, Kioschis P, Poustka A, Meergans K, Doenecke D. Human histone gene organization: nonregular arrangement within a large cluster. Genomics. 1997:40:314-22.

43. Kim M-S, Pinto SM, Getnet D, Nirujogi RS, Manda SS, Chaerkady R, et al. A draft map of the human proteome. Nature. 2014;509:575-81.

44. Mendioroz M, Do C, Jiang X, Liu C, Darbary HK, Lang CF, et al. Trans effects of chromosome aneuploidies on DNA methylation patterns in human Down syndrome and mouse models. Genome Biol. 2015;16:263.

45. Sharma A, Klein SS, Barboza L, Lohdi N, Toth M. Principles Governing DNA Methylation during Neuronal Lineage and Subtype Specification. J Neurosci. 2016;36:1711-22.

46. Henneman P, Bouman A, Mul A, Knegt L, Van Der Kevie-Kersemaekers AM, Zwaveling-Soonawala $\mathrm{N}$, et al. Widespread domain-like perturbations of DNA methylation in whole blood of Down syndrome neonates. PLoS One. 2018;13:e0194938.

47. Garagnani P, Franceschi C, Salvioli S, Di Blasio AM, Fraga M, Castellani G, et al. Identification of a DNA methylation signature in blood cells from persons with Down Syndrome. Aging. 2014;7:82-96.

48. Manley NR, Capecchi MR. Hox group 3 paralogous genes act synergistically in the formation of somitic and neural crest-derived structures. Dev Biol. 1997:192:274-88.

49. Vanderkraats ND, Hiken JF, Decker KF, Edwards JR. Discovering highresolution patterns of differential DNA methylation that correlate with gene expression changes. Nucleic Acids Res. 2013;41:6816-27.

50. Edgar R, Tan PPC, Portales-Casamar E, Pavlidis P. Meta-analysis of human methylomes reveals stably methylated sequences surrounding $\mathrm{CpG}$ islands associated with high gene expression. Epigenetics Chromatin. 2014;7:28.

51. Choe S-K, Vlachakis N, Sagerstrom CG. Meis family proteins are required for hindbrain development in the zebrafish. Development. 2002;129:585-95.

52. Dierssen M. Down syndrome: the brain in trisomic mode. Nat. Rev. Neurosci. 2012;13(12):844-58.

53. Klemm SL, Shipony Z, Greenleaf WJ. Chromatin accessibility and the regulatory epigenome. Nat Rev Genet. 2019;20:207-20.

54. Gibbons RJ, McDowell TL, Raman S, O'Rourke DM, Garrick D, Ayyub H, et al. Mutations in ATRX, encoding a SWI/SNF-like protein, cause diverse changes in the pattern of DNA methylation. Nat Genet. 2000;24:368-71.

55. Amir RE, Van den Veyver IB, Wan M, Tran CQ, Francke U, Zoghbi HY. Rett syndrome is caused by mutations in X-linked MECP2, encoding methylCpG-binding protein 2. Nat Genet. 1999;23:185-8.

56. Schuster J, Laan L, Klar J, Jin Z, Huss M, Korol S, et al. Transcriptomes of Dravet syndrome iPSC derived GABAergic cells reveal dysregulated pathways for chromatin remodeling and neurodevelopment. Neurobiol Dis. 2019;132:104583.

57. Suzuki MM, Bird A. DNA methylation landscapes: provocative insights from epigenomics. Nat Rev Genet. 2008;9:465-76.

58. Cassandri M, Smirnov A, Novelli F, Pitolli C, Agostini M, Malewicz M, et al. Zinc-finger proteins in health and disease. Cell death Discov. 2017;3:17071. 
59. Ladomery M, Dellaire G. Multifunctional zinc finger proteins in development and disease. Ann Hum Genet. 2002;66:331-42.

60. Havis E, Coumailleau P, Bonnet A, Bismuth K, Bonnin M-A, Johnson R, et al. Sim2 prevents entry into the myogenic program by repressing MyoD transcription during limb embryonic myogenesis. Development. 2012;139: 1910-20.

61. Rachidi M, Lopes C, Charron G, Delezoide A-L, Paly E, Bloch B, et al. Spatial and temporal localization during embryonic and fetal human development of the transcription factor SIM2 in brain regions altered in Down syndrome. Int J Dev Neurosci. 2005;23:475-84.

62. Jin S-G, Kadam S, Pfeifer GP. Examination of the specificity of DNA methylation profiling techniques towards 5-methylcytosine and 5hydroxymethylcytosine. Nucleic Acids Res. 2010;38:e125.

63. Tahiliani M, Koh KP, Shen Y, Pastor WA, Bandukwala H, Brudno Y, et al. Conversion of 5-methylcytosine to 5-hydroxymethylcytosine in mammalian DNA by MLL partner TET1. Science. 2009;324:930-5.

64. Kriaucionis S, Heintz N. The nuclear DNA base 5-hydroxymethylcytosine is present in Purkinje neurons and the brain. Science. 2009;324:929-30.

65. Maity B, Sheff D, Fisher RA. Immunostaining: detection of signaling protein location in tissues, cells and subcellular compartments. Methods Cell Biol. 2013;113:81-105.

66. Aryee MJ, Jaffe AE, Corrada-Bravo H, Ladd-Acosta C, Feinberg AP, Hansen $K D$, et al. Minfi: a flexible and comprehensive Bioconductor package for the analysis of Infinium DNA methylation microarrays. Bioinformatics. 2014;30: 1363-9.

67. Koressaar T, Remm M. Enhancements and modifications of primer design program Primer3. Bioinformatics. 2007;23:1289-91.

\section{Publisher's Note}

Springer Nature remains neutral with regard to jurisdictional claims in published maps and institutional affiliations.

Ready to submit your research? Choose BMC and benefit from:

- fast, convenient online submission

- thorough peer review by experienced researchers in your field

- rapid publication on acceptance

- support for research data, including large and complex data types

- gold Open Access which fosters wider collaboration and increased citations

- maximum visibility for your research: over $100 \mathrm{M}$ website views per year

At $\mathrm{BMC}$, research is always in progress.

Learn more biomedcentral.com/submissions 